\title{
Autoimmune Diabetes Associated With Pembrolizumab: A Review of Published Case Reports
}

\author{
Anmol Cheema ${ }^{a}$, Bhaktidevi Makadia ${ }^{a}$, Tejas Karwadia ${ }^{a}$, Ravneet Bajwa ${ }^{a}$, \\ Mohammad Hossain ${ }^{\mathrm{a}}$
}

\begin{abstract}
The utility of immunotherapy, such as pembrolizumab, is becoming essential in the treatment of certain cancers. Pembrolizumab works through binding of programmed cell death 1 receptor that blocks the binding of the programmed cell death ligand 1 and is commonly used in non-small cell lung cancer and melanoma. Pembrolizumab has been reported to be associated with multiple adverse reactions such as pneumonitis, colitis, hepatitis, hypophysitis, hyperthyroidism, hypothyroidism, nephritis, and type 1 diabetes; however, pembrolizumab causing type 1 diabetes was only reported in $0.1 \%$ of the patients in clinical trials. A review of the literature generated 1,001 unique citations of which six reported cases of autoimmune diabetes associated with pembrolizumab were selected and compared. Review of the cases showed no sexual predilection and the average age of onset was 58 years old. The majority of the patients were treated for melanoma (5/6 cases), initially presented with diabetic ketoacidosis (4/6 cases), and had at one point taken ipilimumab ( $4 / 6$ cases). There was no association found between the number of treatments received and the development of diabetes. With the increasing use of pembrolizumab in cancer treatment regular blood glucose monitoring during treatment, especially in patients who had also taken ipilimumab, may prevent the onset of this life-threatening complication.
\end{abstract}

Keywords: Immunotherapy; Pembrolizumab; Autoimmune diabetes; Diabetic ketoacidosis

\section{Introduction}

Immunotherapy has become an important tool in our goal for individualized cancer therapy. The immune system has the ability to distinguish self from non-self and can mount an attack on the non-self-tissue $[1,2]$. The rationale of use of immunotherapy in cancer is its ability to recognize "altered

Manuscript submitted January 23, 2018, accepted February 26, 2018

aJersey Shore University Medical Center, Neptune, NJ, USA

${ }^{b}$ Corresponding Author: Anmol Cheema, Department of Medicine, Jersey Shore University Medical Center, 1945 State Route 33, Neptune, NJ 07753, USA. Email: Anmol.Cheema@hackensackmeridian.org

doi: https://doi.org/10.14740/wjon1085w self" tissue and use the medications to enhance or induce the body's own immune system against cancer cells. Among the most promising approaches for this is the blockage of immune checkpoints $[3,4]$. Immune checkpoints are inhibitory pathways that keep the immune system in check to maintain self-tolerance and prevent autoimmunity $[3,4]$. Blocking of immune checkpoints by various immune-modulating therapies leads to immune activation against tumor cells [4]. Currently, the most common mechanism is to use targeted monoclonal antibodies against immune checkpoints which inhibit T-cell activation [4]. Pembrolizumab is a monoclonal antibody that blocks inhibitory ligand of programmed cell death 1 (PD-1) receptor on T-cells [3].

Pembrolizumab was approved by FDA in 2014 for advanced melanoma and in 2015 for metastatic non-small cell lung cancer (NSCLC) [1]. Despite impressive survival benefits of pembrolizumab in patients with melanoma and NSCLC, its use can be hampered by occurrence of serious adverse events related to excessive immune activation [5]. Most common adverse events from the data pooled from clinical trials include pneumonitis, colitis, hepatitis, hypophysitis, hyperthyroidism, hypothyroidism and nephritis [1,5]. Type 1 diabetes was reported in only $0.1 \%$ of the patients in clinical trials [1]. We conducted a review of case reports of autoimmune diabetes associated with pembrolizumab to study the patient characteristics, possible predisposing factors, presentation, management and clinical outcomes of this rare adverse event.

\section{Literature Search}

We searched two databases: PubMed Central and the Cochrane Library, with no language restrictions up to October 2016. Broad search terms were used such as pembrolizumab, Keytruda, autoimmune diabetes, type 1 diabetes mellitus, antiPD1, PD-L1 inhibitors, programmed cell death 1, MK-3475, endocrine toxicities and adverse events with immunotherapy. All eligible publications were individually reviewed. At first, the titles and abstracts were reviewed and then for relevant articles the full text was reviewed. Original case reports reporting autoimmune diabetes (type 1 diabetes) in patients with cancer, following treatment with pembrolizumab (Keytruda) were included. A total of 1,001 unique citations were initially retrieved. We found six case reports relevant after viewing the full publications.

To evaluate the quality of case reports, we used the 


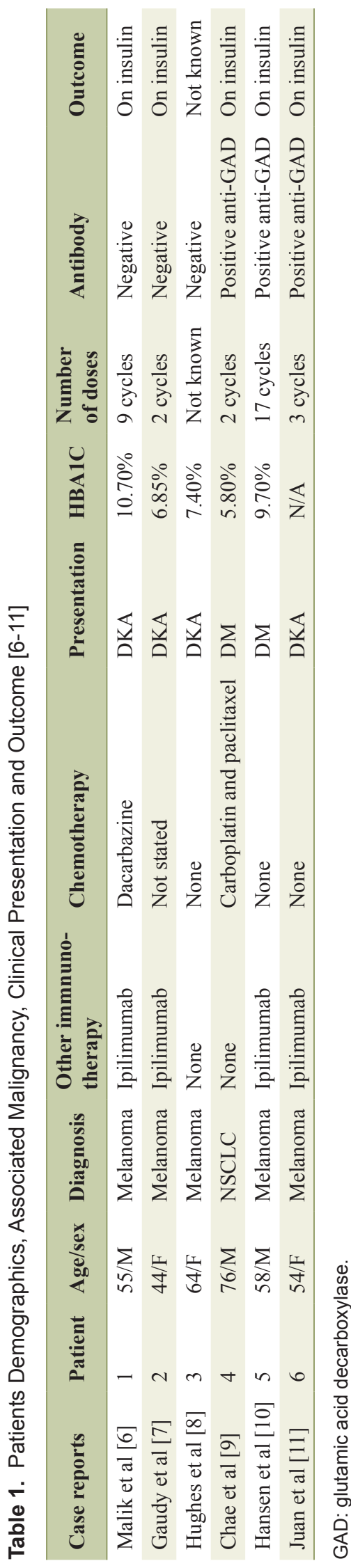

guidelines for publishing adverse events recommended by International Society for Pharmacoepidemiology (ISPE) and International Society of Pharmacovigilance (ISoP). The case reports were assessed on basis of 1) The relevance of the title to reported information; 2) Adequate description of the patient (past medical history, demographics, and laboratory abnormalities); 3) Description of autoimmune diabetes/type 1 diabetes and its outcome. Our study is limited by the information available in the original reports.

\section{Literature Retrieved}

The six cases were reviewed and there salient points were compiled in Table 1 [6-11]. There was no sex predilection with three females and three males affected and the average age at presentation was 58 years old. The majority of patients affected had the initial diagnosis of melanoma although it is unknown if this is significant. Most of the cases presented with diabetic ketoacidosis (DKA) (4/6 cases), and all of them were managed by intravenous (IV) fluids and insulin as the standard practice. Steroids which are used to treat most immune toxicities caused by pembrolizumab were not used in any of the reported cases except patient 4 . However, prednisone was stopped after 25 days when no improvement was seen in that patient's glycemic control. Another important finding is that in most of the cases, patients have been previously on ipilimumab ( $4 / 6$ cases) and then switched to pembrolizumab for non-response or intolerance. The hemoglobin A1C (HBA1C) for the cases varied from normal to elevated. Although, all cases that presented with DKA had elevated HBA1C indicating that the patient must have had elevated glucose for a while before presentation. Presentation in most of the cases was 1 to 4 weeks after the last dose of pembrolizumab; however there was no significant relationship identified between the number of doses and onset of symptoms.

\section{Discussion}

Immunotherapy has revolutionized the management of cancer. With advancing interest and research in this field its use will further increase in various types of malignancies. Currently pembrolizumab is approved for melanoma, NSCLC, recurrent/metastatic head and neck cancer and refractory classical Hodgkin's lymphoma $[1,5]$. Pembrolizumab is a monoclonal antibody that blocks inhibitory ligand of PD-1 receptor on T-cells [3]. Programmed cell death ligand 1 (PD-L1) is a molecule present on the cell surface which bind to the PD-1 receptors on T-cells $[2,3,8]$. The binding of the PD-L1 and PD-1 prevents the T-cell from releasing its cytokines and destroying the cell with the PD-L1 $[3,8]$. However, the problem comes when the cancer cells also express PD-L1 which protect them being destroyed by T-cells. Therefore, pembrolizumab function of binding to PD-1 receptors on T cells will block the inhibitory association with PD-L1 thereby allowing T-cell to mount an immune response against cancer cells $[1,7]$. The problem is that pembrolizumab is not specific and therefore 
normal, non-cancerous cells will no longer be able to inhibit T-cells which will result in the death of self cells [1, 7]. This is the mechanism for many of the adverse effects caused by pembrolizumab [7].

The mechanism of the production of autoantibodies due to PD-L1 therapy is not well known or well researched. A possible mechanism is due to the destruction of the beta islet cells of the pancreas by host T-cells which are no longer inhibited due to the effects of the PD-L1 therapy. The beta islet cells of the pancreas are the cells responsible for the production and regulation of insulin [2]. In a study performed on mice that were given doses of PD-L1 therapy the histologic findings of the pancreases of the mice that developed diabetes had destruction of the cells in the islets of Langerhans and there was also an increased PD-L1 expression in these islet cells [2]. This shows that the drug acts directly on the beta islet cells of the pancreas and therefore aid in the destruction of the insulin producing cells. The destruction of the beta islet would cause a release of intracellular proteins, such as glutamic acid decarboxylase (GAD). GAD is an enzyme that is found in the beta islet cells of the pancreas and has a role in the decarboxylation of glutamate to gamma-aminobutyric acid (GABA) [2]. The release of these intracellular proteins could trigger a response by the immune system to form autoantibodies such as anti-GAD. The destruction of the beta islet cells due to PD-L1 therapy has similar pathophysiology as that of an autoimmune type 1 diabetic patient. Due to a potential rapid destruction of the beta islet cells this could result in a sudden drop in insulin stores resulting in DKA.

A recent phase II clinical trial showed a response rate of $56 \%$ when pembrolizumab is used to treat advance Merkel cell carcinoma $[1,3]$. In context of increasing indications of pembrolizumab, clinicians and patients must be aware of this rare but potentially life-threatening complication. While common adverse events have been well described in clinical trials (e.g. pneumonitis, colitis, hepatitis, hypophysitis, hyperthyroidism) rare adverse events such as autoimmune diabetes needs to be further studied $[9,10]$. These case reports are a vital clue that can help us understand the presentation, management and clinical outcome of autoimmune diabetes associated with pembrolizumab.

We reviewed the development of autoimmune diabetes in six patients after receiving pembrolizumab. Four out of six patients $(67 \%)$ were previously on ipilimumab which intensifies the immune toxicity of pembrolizumab and can be a possible aggravating factor in precipitating autoimmune diabetes. Interestingly most of the cases presented as DKA (4/6 patients) which highlights that the pathophysiology of this immune toxicity is likely acute. DKA as the first presenting symptom should also alert the clinician about the high mortality of this adverse event. Hence, although rare, new onset diabetes mellitus and potentially DKA as an adverse event should be discussed with the patients before starting this immunotherapy. Frequent blood glucose monitoring or a decreased threshold for glucose monitoring can also be considered in order to recognize new onset diabetes thereby hopefully avoiding DKA as its presenting symptom. Two out of six patients had high risk HLA subtypes which can predispose to type 1 diabetes, although in other cases these HLA subtypes were not tested.
This highlights the fact that further studies need to be done to recognize any predisposing factors or drugs that might lead to autoimmune diabetes associated with pembrolizumab. Almost all the patients, except one continued to be on insulin therapy which emphasizes the fact that unlike other immune toxicities, autoimmune diabetes secondary to pembrolizumab might be irreversible. The use of anti-GAD serology in aiding the diagnosis is not recommended as only half of the patients were positive although this can potentially be used to confirm the diagnosis if positive. Steroids are used to treat most immune toxicities with pembrolizumab however; their use has not been established in treatment of autoimmune diabetes [11]. The ideal management of this complication is yet to be determined and currently there are no recommended treatments other than insulin therapy.

\section{Conclusions}

Autoimmune diabetes and possible resultant DKA are rare but life-threatening adverse events associated with pembrolizumab. Regular blood glucose monitoring can help clinicians to diagnose new onset diabetes and to avoid complication of DKA. Currently there are no recognized predisposing factors or any dose dependent toxicity that can precipitate autoimmune diabetes in patients using pembrolizumab. The use of steroids to treat autoimmune diabetes is controversial and the ideal management of this complication is yet to be determined. Currently, aside from insulin therapy there are no exact guidelines.

In the context of increasing indications of pembrolizum$\mathrm{ab}$, clinicians should be aware of this rare but potentially lifethreatening complication. Similarly, patient should also be educated about the potential side effect of new onset diabetes. This review also emphasizes the need of further studies to confirm the association of pembrolizumab with autoimmune diabetes. Guidelines need to be set up to identify potential predisposing factors, ideal monitoring and management of this rare adverse event.

\section{References}

1. Abdel-Wahab N, Shah M, Suarez-Almazor ME. Adverse events associated with immune checkpoint blockade in patients with cancer: a systematic review of case reports. PLoS One. 2016;11(7):e0160221.

2. Ansari MJ, Salama AD, Chitnis T, Smith RN, Yagita H, Akiba H, Yamazaki T, et al. The programmed death-1 (PD-1) pathway regulates autoimmune diabetes in nonobese diabetic (NOD) mice. J Exp Med. 2003;198(1):6369.

3. Faghfuri E, Faramarzi MA, Nikfar S, Abdollahi M. Nivolumab and pembrolizumab as immune-modulating monoclonal antibodies targeting the PD-1 receptor to treat melanoma. Expert Rev Anticancer Ther. 2015;15(9):981993.

4. Guleria I, Gubbels Bupp M, Dada S, Fife B, Tang Q, Ansari MJ, Trikudanathan S, et al. Mechanisms of PDL1- 
mediated regulation of autoimmune diabetes. Clin Immunol. 2007;125(1):16-25.

5. Chan MM, Kefford RF, Carlino M, Clements A, Manolios N. Arthritis and tenosynovitis associated with the anti-PD1 antibody pembrolizumab in metastatic melanoma. J Immunother. 2015;38(1):37-39.

6. Humayun MA, Poole R. A case of multiple immune toxicities from Ipilimumab and pembrolizumab treatment. Hormones (Athens). 2016;15(2):303-306.

7. Gaudy C, Clevy C, Monestier S, Dubois N, Preau Y, Mallet S, Richard MA, et al. Anti-PD1 pembrolizumab can induce exceptional fulminant type 1 diabetes. Diabetes Care. 2015;38(11):e182-183.

8. Hughes J, Vudattu N, Sznol M, Gettinger S, Kluger H, Lupsa B, Herold KC. Precipitation of autoimmune dia- betes with anti-PD-1 immunotherapy. Diabetes Care. 2015;38(4):e55-57.

9. Chae YK, Chiec L, Mohindra N, Gentzler R, Patel J, Giles F. A case of pembrolizumab-induced type-1 diabetes mellitus and discussion of immune checkpoint inhibitorinduced type 1 diabetes. Cancer Immunol Immunother. 2017;66(1):25-32.

10. Hansen E, Sahasrabudhe D, Sievert L. A case report of insulin-dependent diabetes as immune-related toxicity of pembrolizumab: presentation, management and outcome. Cancer Immunol Immunother. 2016;65(6):765-767.

11. Martin-Liberal J, Furness AJ, Joshi K, Peggs KS, Quezada SA, Larkin J. Anti-programmed cell death-1 therapy and insulin-dependent diabetes: a case report. Cancer Immunol Immunother. 2015;64(6):765-767. 